David V. Beard, PhD • Bradley M. Hemminger, MS • J. Randolph Perry, MD • Matthew A. Mauro, MD

Keith E. Muller, PhD • David M. Warshauer, MD • Michael A. Smith, MD • Alexander J. Zito, MD

\title{
Interpretation of CT Studies: Single-Screen Workstation versus Film Alternator ${ }^{1}$
}

A prototype single-screen workstation with a $2,048 \times 2,560$-pixel highbrightness monitor, 0.11 -second image display time, and simple ergonomic design was compared to a conventional horizontal film alternator in diagnostic interpretation of chest computed tomography (CT) studies. Four radiologists used either the workstation or film alternator in interpretation of studies obtained in 10 patients. A counterbalanced withinsubject repeated measures experimental design was used. Response times were analyzed for both methods of interpretation. Grades of excellent, acceptable, and unacceptable were assigned by a blinded "grader" to reports of the radiologists. The average time needed for an interpretation at the workstation was $5.65 \mathrm{~min}$ utes. No interpretations were graded unacceptable. Retrospective power analysis showed that 16 observers rather than four would have been required to show that use of the workstation was faster than the alternator. With this $95 \%$ confidence interval, the workstation interpretation time is clinically equivalent to that with the alternator. These data show that this type of workstation has practical application in interpretation of CT, magnetic resonance imaging, and ultrasound studies.

Index terms: Computed tomography (CT), image display and recording - Diagnostic radiology, observer performance, 47.1211, 60.1211 • Images, display - Images, interpretation - Picture archiving and communication system

Radiology 1993; 187:565-569
$\mathbf{P}^{\prime}$ ICTURE archiving and communication systems (PACS) hold the promise of reduced costs $(1,2)$ and improved logistics through reduction in film expenses, simultaneous access by multiple radiologists to the same imaging study, elimination of lost examinations, and the potential for cost-effective access to various image processing techniques $(3,4)$. This is particularly true for modalities such as computed tomography (CT), which are inherently digital and for which multiple small images completely fit onto available monitors. These potential benefits, however, have awaited design of a workstation that can facilitate fast and accurate interpretations of CT studies (5).

A number of researchers have evaluated various workstations for interpretation of CT and magnetic resonance (MR) imaging studies. All of these used one to eight $1,024 \times 1,024$ pixel monitors with image display times ranging from 1.5 to 26 seconds. Foley et al (6) observed reduced accuracy, likely caused by the slow 7.5-26second image display time of the commercial workstation they were evaluating. In general, however, other studies (7-11) have concluded that with a good quality monitor, appropriate window settings, and acceptable computer-interpreter interaction, use of a PACS workstation can provide the same interpretation accuracy as a film alternator.

Interpretation time is another matter. In most of these studies, time with workstations was found to be considerably longer than with a film alternator, with radiologists often taking

\footnotetext{
' From the Departments of Radiology (D.V.B., B.M.H., J.R.P., M.A.M., D.M.W., M.A.S., A.J.Z.), Computer Science (D.V.B.), Biostatistics (K.E.M.), and Surgery (M.A.M.), School of Medicine, University of North Carolina, 509 Old Infirmary Rd, Chapel Hill, NC 27599-7510. Received August 31, 1992; revision requested October 21; revision received December 21; accepted December 29. Supported in part by NIH grant no. RO1 CA44060. Address reprint requests to D.V.B.

RSNA, 1993
}

more than twice as long to interpret a study, although Straub et al (11) found two display modes-one with two high-speed 2,000 $\times 2,000$-pixel monitors, and the other with a highspeed cine or sequential display-to be as fast as film for an abdominal mass detection task involving completion of a computerized findings form as part of a receiver operating characteristic study.

Fast as well as accurate interpretation is essential if a CT workstation is to be clinically effective. To this end, we constructed a prototype workstation called FilmStrip, by using a single 2,048 $\times$ 2,560-pixel high-brightness monitor, a new interface design with a simple computer-human interaction, and an extremely fast image display time. FilmStrip is designed for interpretation of CT, ultrasound (US), and MR imaging studies, which involve a large number of small images.

A time and motion study (12) suggested that image display area, image display time, and a simple interaction are the most important factors in interpretation speed with a workstation. Because of this, we expected FilmStrip to facilitate faster CT scan interpretation than previous electronic workstations. A simplified time and motion analysis $(13,14)$ of an example may be helpful. A previous prototype CT workstation of ours (FilmPlane [8]) used a single $1,024 \times$ 1,024-pixel monitor to display four full-resolution CT images in $1.5 \mathrm{sec}$ onds. Each image display operation displayed a new row containing two CT images. Observation of radiologists using FilmPlane to interpret complex CT studies indicated that on average, 49 image display operations were used to interpret complex 40section chest CT studies. Since each

Abbreviation: $\mathrm{PACS}=$ picture archiving and communication system. 
image display operation required 1.5 seconds, the radiologist spent an average of 73 seconds (49 operations $\times 1.5$ seconds) waiting for images to be displayed during an interpretation.

FilmStrip, on the other hand, allows simultaneous display of 12 full-resolution images, rather than the four that the early workstation could manage. Since many more images are displayed at the same time, the radiologist needs only about 18 image display operations to view the entire study during the interpretation. Also, since the images are displayed in 0.11 second, the radiologist spends an average of only 2 seconds (18 operations $\times 0.11$ second) waiting for images to be displayed during the interpretation, for a savings of over 70 seconds per study when compared with FilmPlane. Additionally, the FilmStrip interaction design requires fewer button presses and other hand motions, which results in even faster interaction times.

We conducted an experiment to evaluate the hypothesis that the interpretation time of chest CT studies with the FilmStrip workstation is clinically equivalent to that with a film alternator.

\section{MATERIALS AND METHODS}

\section{Equipment}

Studies were interpreted with a conventional horizontal film alternator and FilmStrip. This prototype workstation uses a single $28 \times 35-\mathrm{cm}, 2,048 \times 2,560$-pixel, 72 $\mathrm{Hz}$, noninterlaced monitor (Megascan Technology, Littleton, Mass) providing $50 \mathrm{ft}-\mathrm{L}$ of brightness at the center of the screen. This monitor has five times more pixels than a conventional $1,024 \times 1,204-$ pixel display. The monitor is mounted inside a dark, nonglare cabinet, in portrait orientation, with a vertical monitor surface, and with its center at average eye level. This monitor uses a $2,048 \times 2,560$ pixel video buffer and a 4,096 $\times$ 4,096-pixel frame buffer all connected with a VME bus to a workstation (model 4/370; Sun Microsystems, Mountain View, Calif). The frame buffer can contain up to 4012 -bitper-pixel CT images. A special bus connecting the high-speed frame buffer to the video buffer allows any number of images in the frame buffer to be windowed according to intensity, increased or decreased in size, copied to the video buffer, and displayed on the monitor in $0.11 \mathrm{sec}-$ ond. This near-instant image display time is slightly faster than the human reaction time to press a keyboard button.

The design of FilmStrip uses the metaphor $(15,16)$ of a single-panel vertical alternator in which the images are arrayed in a long vertical "filmstrip" with "up" and "down" scroll buttons to control movement (Fig 1). While the $2,048 \times 2,560$ pixel monitor can display simultaneously 20 full-resolution $512 \times 512 \mathrm{CT}$ images, preliminary observer testing indicated that the radiologists prefer images that are the same physical size as film. Interpolation was used to enlarge the images, resulting in $640 \times 640$-pixel, $9 \times 8$-cm images, with 12 images simultaneously displayed in a three column by four row filmstrip. A somewhat smaller 2,000 × 1,600-pixel monitor also would allow display of 12 full-resolution images.

The workstation keyboard is configured into a five-button control panel, with buttons for up and down functions as well as three preset intensity windows (Fig 2). Each operation scrolls the filmstrip up or down by two rows, or half of the display, which allows an image to be compared with all of its neighboring images. Since the majority of CT interaction involves scrolling, we applied the principle of large buttons $(17,18)$ and used the space bar for down and all the other typewriter keys for up. Lung, soft-tissue, and bone preset intensity windows are provided through three function keys at the top of the keyboard. These intensity window settings were fixed for all cases. Since preset intensity windows must be used with an alternator and film, we chose to not provide dynamic intensity windowing, thus allowing a controlled comparison of the workstation and alternator.

\section{Observers and Cases}

Four board-certified radiologists experienced with chest $C T$ participated in the study. All observers were less than 45 years old and had experience with computer word processors.

Eight studies with multiple abnormal findings at chest CT and two studies without abnormalities were selected by a radiologist who was not an observer. Findings among the abnormal cases were distributed as follows: mediastinal or hilar masses or masses bordering the heart $(n=4)$, pleural effusions $(n=2)$, parenchymal infiltrate $(n=1)$, esophageal narrowing $(n=1)$, and positive findings at cytologic evaluation of sputum $(n=1)$. An additional chest CT case was used for training the radiologists to use the workstation. All studies had been obtained with the same CT scanner (2060; Technicare [GE Medical Systems], Milwaukee, Wis), with an average of 37 sections per case. The CT scout view was included in the upper left-hand corner of the filmstrip. To control for the effect of case on outcome, the 10 cases were paired for difficulty and divided into two equal groups. The original CT requisition form, typically including patient history and the referring physician's clinical question, was obtained for each case. The original films were used for alternator viewing.

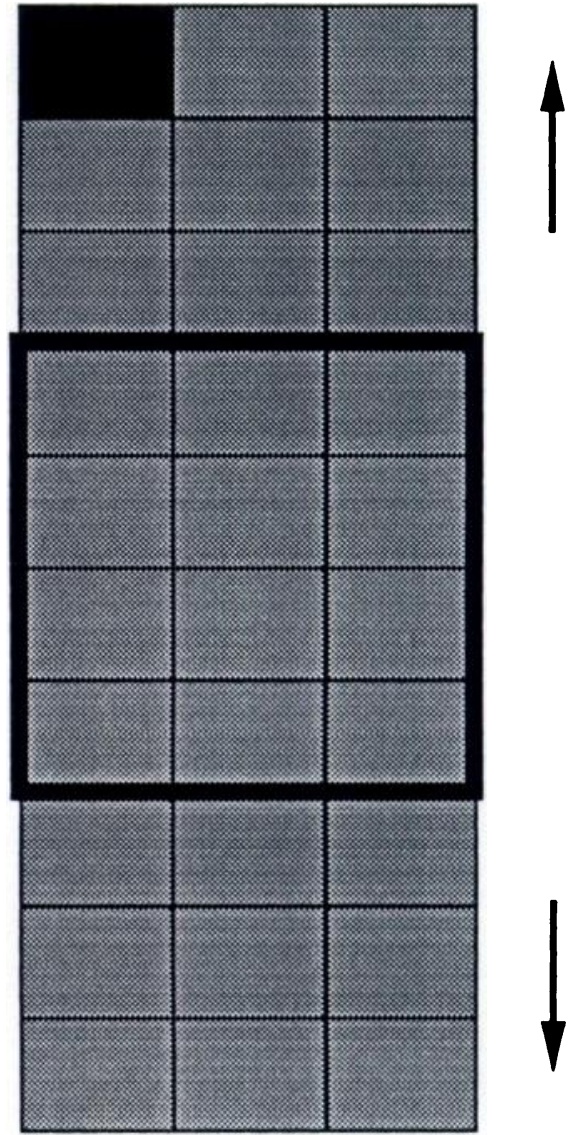

Figure 1. FilmStrip metaphor.

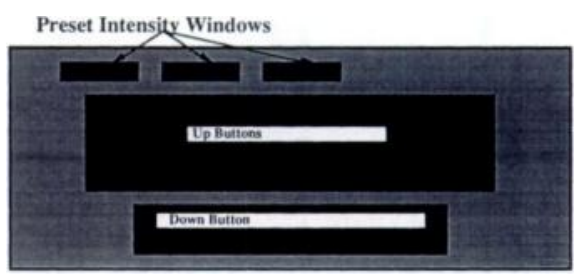

Figure 2. FilmStrip control panel, based on the Sun workstation keyboard.

\section{Experimental Design and Procedure}

A counterbalanced, within-subject experimental design was used, with each observer reading one group of cases by using the film alternator and the other group using the FilmStrip in separate sessions several weeks apart. Each case was read exactly once by each observer, and each case was read the same number of times with the workstation and film alternator. Case presentation, observer, and method were controlled. Two observers started with the workstation and progressed to the alternator, while the others started with the film alternator.

Film alternator sessions were conducted in clinical reading rooms with dimmed lighting during inactive periods. One hour was typically required to interpret the cases and gather the observer's verbal comments. Care was taken to eliminate interruptions and distractions. Observers 
Table 1

Average Interpretation Time

\begin{tabular}{|c|c|c|c|c|}
\hline \multirow[b]{2}{*}{ Observer } & \multicolumn{3}{|c|}{ Average Interpretation Time (min), by Method } & \multirow{2}{*}{$\begin{array}{l}\text { Average of Film } \\
\text { and Workstation } \\
(\mathrm{min})\end{array}$} \\
\hline & Workstation & Film & Prehung Film* & \\
\hline 1 & 4.12 & 3.75 & 2.63 & 3.93 \\
\hline 2 & 7.17 & 7.25 & 6.35 & 7.21 \\
\hline 3 & 4.13 & 5.97 & 4.60 & 5.05 \\
\hline 4 & 7.20 & 7.85 & 6.47 & 7.52 \\
\hline Average & 5.65 & 6.21 & 5.01 & \\
\hline
\end{tabular}

were instructed to work as quickly as possible to produce a report of clinically acceptable quality. For each case, the observer was given a patient film folder containing the single CT chest study and a card containing the relevant requisition information. Films in the patient folder were in the correct order. Observers loaded the films onto the alternator, viewed the images, and dictated the report by using a familiar dictation machine. We used a dictated report rather than the "findings form" typically used for receiver operating characteristic studies, because a findings form can augment human working memory (19) and distort the user's behavior and interpretation time. FilmStrip sessions were conducted in a controlled environment (20). After the dictation device, seat adjustment, and keyboard and monitor placement were checked, the five-button interaction was demonstrated, and the observer trained for 3 to $4 \mathrm{~min}$ utes. The monitor was initially blocked with a large piece of cardboard for each trial. To start each trial, the experimenter displayed the appropriate study on the monitor, removed the cardboard and allowed the observer to see the images, and gave the observer the requisition information. The observer then scrolled back and forth through the images, changed intensity windows as needed, and dictated a report.

\section{Data Collection}

Verbal protocol-that is, verbal comments made by the observer during the experiments-was collected throughout the study. At the end of each session, the experimenter conducted an unstructured interview to determine the observer's feelings and opinions with regard to workstation versus film alternator interpretation. Besides videorecording the postsession review and analysis, the experimenter also used a stopwatch to measure the time used to load the films onto the alternator, the time to file the films back into the patient folder, and the total time of the interpretation, with precision of about $1 \mathrm{sec}$ ond.

In a well-designed PACS environment, the radiologist should not have to wait while the workstation locates, moves, and displays the required case. Avoiding this waiting time can be accomplished either by the workstation predicting which case will be needed next and "prefetching" it (21) or by using an extremely high-speed image storage device and network to transmit the study in a few seconds (2). Because the radiologist should not have to wait for images to be loaded into a welldesigned PACS workstation, the time to load the images into the workstation was not included in the interpretation time for this experiment. Whether film load and unload time should be included in film interpretation time depends on the operational characteristics of the clinical environment of the reader. We report both total film interpretation time (including the time to load and unload the films) as well as prehung film times (without the time to load and unload the films) to allow the reader to make institutionally specific comparisons.

\section{Data Analysis}

Exploratory analysis was used to compare identity, logarithmic, and inverse transformations of response times to ensure that the distribution was acceptably Gaussian. The distribution of the untransformed data was judged to be the best. Hence, response times are analyzed throughout. The univariate approach to repeated measures analysis of variance (22) was conducted on the total time, with trial (numbers 1 through 5) and method (film vs workstation) as repeated factors. Appropriate confidence intervals were created to assess uncertainty of estimates.

We measured interpretation accuracy to ensure valid interpretation times. Accuracy of the dictated reports was analyzed by a radiologist "grader" blinded to observer and interpretation method. All four reports as well as the requisition form and original films were available. The transcribed reports were evaluated as follows. A list of findings was generated for each report. Then, a findings list was generated for each case, on the basis of the patient record, radiologic reports, and the films and images. The findings for each case were further divided into "critical findings," which would affect patient care decisions, "relevant findings" related to the clinical question contained in the requisition form, and additional "noncritical" findings. Finally, the grader examined each report and assigned one of the following overall grades: excellent-no erro- neous findings, all critical findings, all relevant findings, and a rich detail of noncritical findings; acceptable-no erroneous findings, all critical findings, and all relevant findings; or unacceptable-an erroneous finding, a missed critical finding, or a missed relevant finding.

\section{RESULTS}

\section{Data}

The average time needed at the workstation to interpret the CT studies was 5.65 minutes (Table 1). The total film time (with loading and unloading) averaged 6.21 minutes, while prehung film (without loading and unloading) averaged 5.01 minutes. Observers 2, 3, and 4 were faster with the workstation than with film, including loading and unloading time. Observer 1 was faster with film.

Analysis of total time yielded three nonsignificant tests: $(a)$ method by trial $(\mathrm{F}=1.56$ on 4 and $12 d f$ with $P=.25$, Geisser-Greenhouse epsilon of .47$),(b)$ trial $(\mathrm{F}=0.66$ on 4 and $12 d f$ with $P=.63$, Geisser-Greenhouse epsilon of .30$)$, and $(c)$ method $(F=1.38$ on 1 and $3 \mathrm{df}$ with epsilon automatically 1.0). The small number of observations may have limited the sensitivity of the design. Hence, a confidence interval was created for the mean difference between the method total times. The mean difference of the four observations (averaging across the nonsignificant trial factor) was 0.56 minutes, with a standard deviation of 0.95 and a standard error of 0.47 . For the Student $t$ statistic with $3 d f$, a $95 \%$ confidence interval for the mean difference between workstation and total film is therefore $0.56 \pm 0.47$ (2.353), or equivalently, in minutes $(-0.55$, 1.66). Retrospective power analysis indicates that with the same observed values, 16 observers rather than four would have been required to show that the workstation was faster than film at $95 \%$ confidence.

Prehung film times also were compared to workstation total time. The analysis yielded three nonsignificant tests: (a) method by trial ( $\mathrm{F}=1.60$ on 4 and $12 d f$ with $P=.24$, GeisserGreenhouse epsilon of .47), (b) trial $(\mathrm{F}=0.62$ on 4 and 12 df with $P=.66$, Geisser-Greenhouse epsilon of .30), and $(c)$ method $(F=2.51$ on 1 and $3 d f$ with epsilon automatically 1.0 ). The mean difference of the four observations for prehung film time minus workstation total time (averaging across the nonsignificant trial factor) was -0.64 minutes, with a standard deviation of 0.81 and a standard error of 0.40 . For $t$ with $3 d f$, a $95 \%$ confi- 
dence interval for the mean difference between total workstation time and prehung film time is therefore -1.58 \pm 0.40 (2.353), or equivalently, in minutes, $(-1.58,0.30)$.

In short, we are $95 \%$ confident that the FilmStrip workstation is no more than 1 minute and 40 seconds faster than film with loading and unloading and no more than 33 seconds slower. Furthermore, we are $95 \%$ confident that the workstation is no more than 18 seconds faster than prehung film and no more than one minute and 35 seconds slower.

Interpretation accuracy with both film and workstation was nearly identical, with no unacceptable reports dictated for either film or workstation (Tables 2, 3). There was no noticeable difference in performance between the two methods, with film alternator and workstation having almost the same number of excellent and acceptable reports. Technical difficulties with one dictation tape resulted in four dictated reports that could not be analyzed. Learning effect precluded retesting that observer for those cases.

\section{Observations}

Observers expressed feelings of comfort and seemed to feel in control while using the workstation. There was no observed fumbling with the critical up and down buttons. All observers believed that image size and quality were acceptable and that the FilmStrip design provided sufficient display area, speed, and simplicity to be clinically useful. Most important, they all said they would be willing to use a similar system in a clinical setting.

While three observers were faster with the workstation than with film, the fastest observer (observer 1) was faster with film. While being interviewed, observer 1 indicated that he had developed and refined his method of interpreting CT studies with a horizontal alternator over many years. He stated that he expected to be able to interpret CT much faster with FilmStrip after more training, as he would have more time to refine his interpretation behavior to best match the advantages and drawbacks of electronic display.

Several minor problems were encountered. First, observers occasionally had to look down at the keyboard to locate the intensity window keys, increasing the duration of the interpretation. Second, we used a scroll increment of two rows (half screen) per up or down command. The ob-

Table 2

Interpretation Accuracy

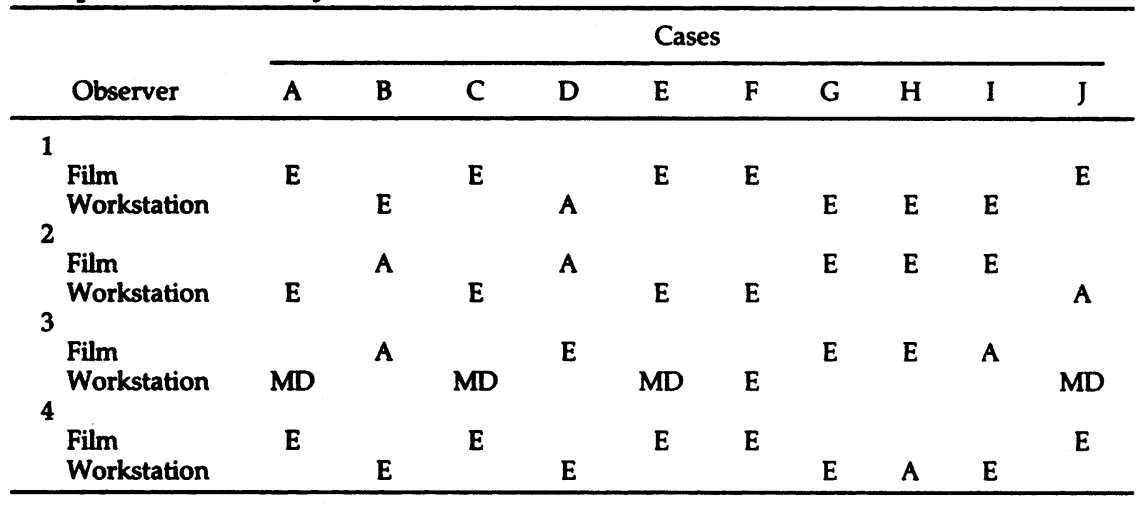

Note. $-\mathrm{A}=$ acceptable, $\mathrm{E}=$ excellent, $\mathrm{MD}=$ missing data point. There were no unacceptable reports.

servers generally managed to maintain spatial context while scrolling and could achieve rapid movement through the examination. The tworow scroll, however, sometimes confused the observers because of the visual similarity of the unnumbered sections in adjacent rows; on several occasions, the observers reinterpreted two rows after scrolling. Third, while all the observers stated that the default windows were adequate, two wanted to adjust the default liver intensity window for several cases.

\section{DISCUSSION}

After 3-4 minutes of training, average interpretation time with the workstation was 34 seconds faster than with the alternator when the time to load and unload the films was included. Given the confidence intervals described in our results, we are comfortable in concluding that workstation interpretation time is clinically equivalent to total film alternator time, including loading and unloading of films.

Interpretation of studies with the workstation averaged 33 seconds slower than with prehung film, with a confidence interval ranging from 18 seconds faster to 1 minute and 35 seconds slower. Thus, it is likely that prehung film would be slightly faster than the workstation in an efficient film operation. Given the work patterns of the typical CT radiologist reading $20-50$ cases per day, not to mention the film handling patterns of the typical clinical operation, we believe that in general, however, the use of a workstation in lieu of prehung film would have no significant impact on daily productivity. Further, we expect reductions in workstation interpretation time after several minor improvements are made to the user interface design and after radiologists receive additional training and be-
Table 3

Summarized Interpretation Accuracy

\begin{tabular}{lccc}
\hline & \multicolumn{3}{c}{ Rating } \\
\cline { 2 - 4 } Method & Excellent & $\begin{array}{c}\text { Ac- } \\
\text { ceptable }\end{array}$ & $\begin{array}{c}\text { Unac- } \\
\text { ceptable }\end{array}$ \\
\hline Film & $16(80)$ & $4(20)$ & 0 \\
Workstation & $13(81)$ & $3(19)$ & 0 \\
\hline
\end{tabular}

Note.-Values in parentheses are percentages.

come more familiar with workstation interaction and learn how best to integrate it into their clinical activity.

Therefore, given the similarity in interpretation times and the logistic advantages of PACS, we believe that an effective PACS with a workstation similar to FilmStrip should be a clinically equivalent alternative to film for clinical chest CT, especially when images are needed in several locations at the same time during patient work-up. Further, we believe that because of the similarity in image interaction, these results can be extended to use in general CT, MR imaging, and US.

\section{Caveats}

Observers.-The observers were sufficiently computer literate to use word processors. It is not clear that similarly rapid acceptance would be found with radiologists who feel uncomfortable interacting with computers, but we would expect similar interpretation times after sufficient training.

Cases.-Each case was read the same number of times for a given method and observer, controlling for the relative effect of case on interpretation time. Absolute interpretation times for both film and workstation, however, were affected by the complex abnormal cases we selected.

Fatigue.-Each interpretation session 
with the workstation lasted less than 1 hour. It is possible that after longer interpretation sessions, mental and eye fatigue of the film alternator and workstation might affect relative performance or comfort.

Single versus multiple examinations.Our interpretation tasks involved only single CT studies, with no comparison of serial studies. A two-monitor version of FilmStrip, however, with each monitor containing a filmstrip of a different study and operated either independently or in a linked way can be used to simultaneously present serial studies.

\section{Implications for Future Workstation Design}

Speed.-Fast image display is essential for a fast interpretation time. A typical interpretation may require from 30 to 60 scrolling operations, so decreasing image display time from 2.0 seconds to 0.1 second could decrease interpretation time by up to 1.9 minutes. Ideally, image display time would be in the 0.1 second range, with no apparent image display lag after a scrolling command.

Screen space.-Previous work (23) with an eye tracker suggests that radiologists need to view simultaneously at least six to eight images in a multipleimage mosaic display for effective single CT study interpretation. Additionally, with more images displayed simultaneously, fewer scroll operations are needed, which further reduces interpretation time. On the other hand, too many monitors may spread the images over too large a physical area and actually slow the interaction. A multipleimage mosaic display of from eight to 12 images per study on one or two monitors with a quick response time may provide an effective balance for interpretation of single studies. A high-speed cine display (11) sequentially showing single images has the potential to greatly reduce screen space requirements for a workstation, but more study is needed before the relative interpretation speed for a primary interpretation task involving report dictation can be determined for the cine mode.

Simplicity.-Our experimental results suggest that extremely simple interaction may be important. As suggested by Brown et al (9), we did not include zooming, general roaming, a magnifying glass, individual image movement, or many other commonly available functions. While these functions may provide some additional utility, they increase overall interpretation time, and they make systems harder to learn and use.

FilmStrip is a prototype developed to focus on the computer-human interaction aspects of $C T$ interpretation, and as such, it omits the essential connections to radiology information systems, hospital information systems, and the general PACS environment. A clinically viable CT workstation might also include dynamic intensity windowing and other image processing functions for occasional use; linear, area, and volume measuring; and cine display, multiplanar reformation, three-dimensional volume rendering, and Hounsfeld unit measuring. A more complex interaction may also be required for a workstation designed to interpret multiple modalities. The integration of this additional function will necessarily increase the complexity of the interaction and possibly increase interpretation time. Further, it is possible that a well-designed, mouse-based interface may be superior to our five-button control panel for a more complex interaction.

While we encountered no critical interpretation quality problems with our general preset intensity windows, verbal comments from the observers indicated the desire for intensity window values set for each particular study. While dynamic intensity windowing by the radiologist is a possible solution, it often takes more than 10 seconds per adjustment, which potentially decreases overall productivity. A clinical operation also can be designed to have technologists preselect intensity windows for each study. The relative cost-effectiveness of these two approaches is beyond the scope of this article.

Cost.-The hardware used for this experiment cost about $\$ 65,000$ in 1992 , and the software development costs of a similar commercial product might be double or triple this expense. Until lower-cost 2,048 $\times 2,560$-pixel monitors become available, their practical radiologic use may be justified only for high-volume modalities such as computed radiography. Thus, for CT and MR imaging alone, a superior workstation might be based on a relatively low-cost prototype workstation that we have built, called FilmStriplet, which uses a standard workstation (Sparc 2; Sun Microsystems) expanded to include $128 \mathrm{Mbytes}$ of main memory, along with two $1,024 \times$ 1,024-pixel monitors. This Sun workstation has a hardware cost of about $\$ 15,000$, although software costs would make a similar commercial CT workstation considerably more expensive. FilmStriplet can display eight full-resolution CT images in about $0.05-0.1$ second and allows rapid scrolling and selection of fixed-intensity windows. Further study is needed, however, before the potential role of FilmStriplet in clinical diagnosis can be established.

Acknowledgments: We thank Joseph Lee, MD, Richard Clark, MD, and Gene Johnston, $\mathrm{PhD}$, for help in preparation of this manuscript.

\section{References}

1. Arenson RL, van der Voorde F, Stevens JF mproved financial management of the radiology department with a microcosting system. Radiology 1988; 166:255-259.

2. Beard DV, Parrish D, Stevenson D. A cost analysis of film image management and four PACS based on different network protocols. J Digit Imaging 1990; 3:108-118.

3. Pizer SM. Psychovisual issues in the display of medical images. In: Hoehne KH, ed. Pictorial information systems in medicine 1985 . Berlin, Germany: Springer-Verlag, 235-250.

4. Pizer SM, Beard DV. Medical image workstations: state of science and technology. J Digit Imaging 1989; 2:185-193.

5. Arenson RL, Chakraborty DP, Seshadri SB Kundel HL. The digital imaging workstation. Radiology 1990; 176:303-315.

6. Foley WD, Jacobson DR, Taylor AJ, et al. Display of CT studies on a two-screen electronic workstation versus a film panel alternator: sensitivity and efficiency among radiologists. Rensitivity and efficiency among

7. Johnston RE, Yankaskas BC, Perry JR, Pizer SM, Delany DJ, Parker LA. Agreement experiments: a method for quantitatively testing new medical image display approaches. Proc SPIE 1990; 1234:621-630.

8. Beard DV. Designing a radiology workstation: a focus on navigation during the interpretation task. J Digit Imaging 1990; 3:152-163. . Brown JJ, Malchow SC, Totty WG, et al. MR examination of the knee: interpretation with multiscreen digital workstation vs hardcopy format. AJR 1991; 157:81-85.

10. Berbaum KS, Franken EA, Honda H, McGuire C, Weiss RR, Barloon T. Evaluation of a PACS workstation for assessment of body CT studies. J Comput Assist Tomogr 1990; 4:853 858 .

11. Straub WH, Gur D, Good WF, et al. Primary CT diagnosis of abdominal masses in a PACS environment. Radiology 1991; 178:739-743.

12. Beard DV, Hemminger BM, Denelsbeck KM, Brown PH. A time-motion comparison of several radiology workstation designs and film. In: Proceedings of the Symposium for film. In: Proceedings of the Symposium for more, Md, 1992. Carlsbad, Calif: Symposia Foundation, 1992; 588-594.

13. Gilbreth FB. Motion study. New York, NY: van Nostrand, 1911.

14. Card SK, Moran TP, Newell A. The psychology of human-computer interaction. Hillsdale NI: Erlbaum, 1983 .

15. Rumelhard D, Norman D. Analogical processes in learning. In: Anderson JR, ed. Cognitive skills and their acquisition. Hillsdale, NJ: Erlbaum, 1981; 335-339.

16. Young $R$. The machine inside the machines: user's models of pocket calculators. Int J ManMachine Studies $1981 ; 15: 51-85$.

17. Fitts PM. The information capacity of the human motor system in controlling the amplitude of movement. J Exp Psychol 1954; 47:381391.

18. Meyer DE, Abrams RA, Kornblum S, Wright CE. Optimality in human motor performance: ideal control of rapid aimed movements. Psychol Rev 1988; 95:340-370.

19. Miller GA. The magical number seven plus or minus two: some limits on our capacity for processing information. Psychol Rev 1956; 63: 81-97.

20. Rogers DC, Johnston RE, Hemminger BM, Pizer SM. Effect of ambient light on electronically displayed medical images as measured by luminance-discrimination thresholds. J Opt Soc Am 1987; 4:976-983.

21. Wendler TH, Grewer R, Monnich KJ, Svensson $H$. Design considerations for multimodality medical image workstations. In: Hohne KH, ed. NATO ASI series. Vol F19, Pictorial information systems in medicine. Berlin, Germany: Springer-Verlag, 1985; 401-420.

22. Kirk RE. Experimental design. Belmont, Calif: Wadsworth, 1982

23. Beard DV, Johnston RE, Toki O, Wilcox C. A study of radiologists viewing multiple computed tomography studies using an eyetracking device. JDigit Imaging 1990; 3:230-237. 